\title{
Research of temperature stress Non-abutment slant-legged rigid frame bridge
}

\author{
Xiuling He \\ ${ }^{1}$ Yunnan Jiaotong College, Kunming 650500, China; \\ 762104710@qq.com
}

Keywords: Non-abutment slant-legged rigid frame brige; temperature stress; girder and diagonal rod

\begin{abstract}
In this paper through the establishment of the Midas model, mainly discusses the non abutment slant leg rigid frame bridge in different position in the temperature change stress changes. The results of the study show that, with the Overall temperature changes, no abutment slant leg rigid frame bridge girder and diagonal rod of the total stress will have a considerable impact.
\end{abstract}

\section{Introduction}

Non-abutment slant-legged rigid frame brige is a new bridge structure developed in recent years at home and abroad. This kind of bridge has the following characteristics: force reasonable and economic use of materials and medium span all can be used, it is generally designed to crosses the river in a single span or multiple span bridge. In particular, its shape is particularly beautiful, coupled with the line is very concise and lively, so that people have the feeling of mechanical beauty.

Yunnan's climate is a big difference in temperature, so it is necessary to analyze the temperature stress of bridge in Yunnan province.

\section{Engineering background}

Agricultural road bridge, the main span of $33 \mathrm{~m}$, design reference period for 100 years, upper structure with variable section slant legged rigid frame bridge, skew angle for 15 degrees, bridge the $25 \mathrm{~m}$ wide, consists of two pieces of $12.5 \mathrm{~m}$ width variable section beam body composition, each piece section floor $9 \mathrm{~m}$ wide, wing plate width $1.75 \mathrm{~m}$ and single beam using a single box double room section, cross edge web thickness is $0.5 \mathrm{~m}$, across the beam height $1.2 \mathrm{~m}$, slant leg support beam at $1.8 \mathrm{~m}$ high, pivot side span beam depth at $1.4 \mathrm{~m}$. Roof thickness $0.25 \mathrm{~m}$, plate thickness from $0.25 \mathrm{~m}$ changes across to cross pivot $0.45 \mathrm{~m}$, girder high from mid span to cross the fulcrum section of the curve is $\mathrm{y}=0.0025558 \mathrm{x}^{2}+1.2(\mathrm{~m})$, plate thickness variation curve is $\mathrm{y}=0.000852 \mathrm{x}^{2}+0.25$ (m), slant leg by $1.2 \mathrm{~m}$ section form, the diagonal rod horizontal tilt angle for 55.43 degrees, inclined leg horizontal tilt angle is 45 degrees.

MIDAS model as shown in Figure 1

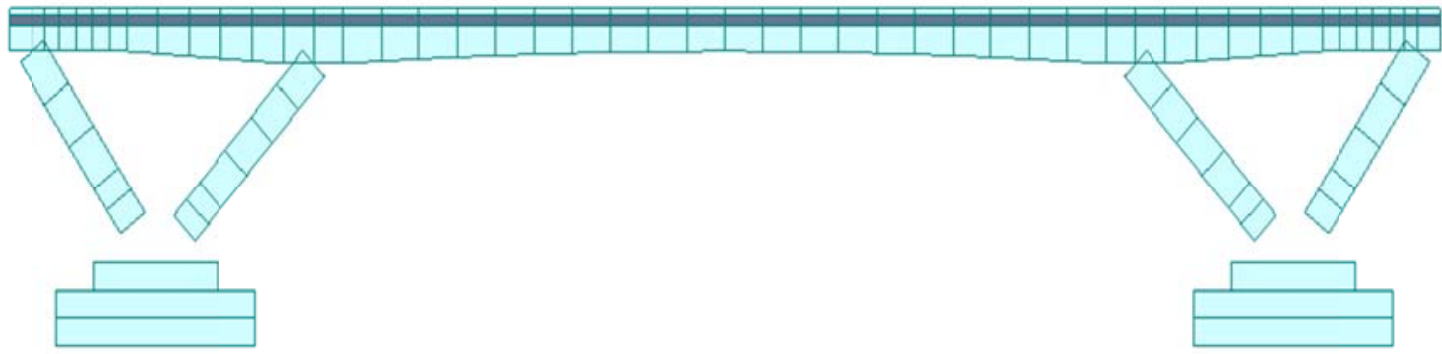

Fig. 1 Model of non-abutment slant-legged rigid frame brige analysis of the Temperature stress 


\section{Overall Effect of temperature difference}

Agricultural road and bridge as an example, due to the seasonal temperature difference, bridge in the operation process of the maximum temperature and the minimum temperature must exist bigger difference, in non abutment slant legged rigid frame bridge is the number of statically indeterminate structure will have larger secondary internal force, selected temperature changes in the structure as shown in the following table:

Table 1 Structure overall temperature change table

\begin{tabular}{|l|r|r|r|r|r|c|}
\hline Overall warming & $10^{\circ} \mathrm{C}$ & $20^{\circ} \mathrm{C}$ & $30^{\circ} \mathrm{C}$ & $40^{\circ} \mathrm{C}$ & $50^{\circ} \mathrm{C}$ & $60^{\circ} \mathrm{C}$ \\
\hline Overall cooling & $-10^{\circ} \mathrm{C}$ & $-20^{\circ} \mathrm{C}$ & $-30^{\circ} \mathrm{C}$ & $-40^{\circ} \mathrm{C}$ & $-50^{\circ} \mathrm{C}$ & $-60^{\circ} \mathrm{C}$ \\
\hline
\end{tabular}

Based on the analysis of the stress state in the bridge's cross section at different temperatures to for the design, construction and operation of this kind of bridge provides the basis.

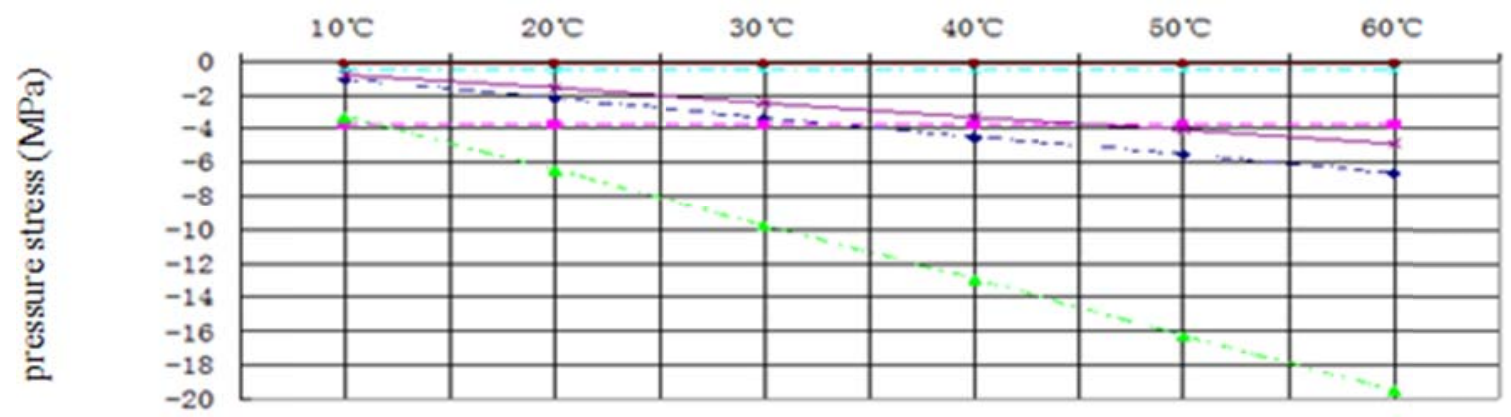

Cross-sectional temperature pressure stress

Temperature pressure stress at the bottom of the side slope Side span cross-sectional temperature pressure stress
Cross-sectional weight pressure stress

The gravity pressure stress of the bottom section of the side slope Side cross-sectional weight pressure stress Fig 2 the bridge pressure stress when the overall warming

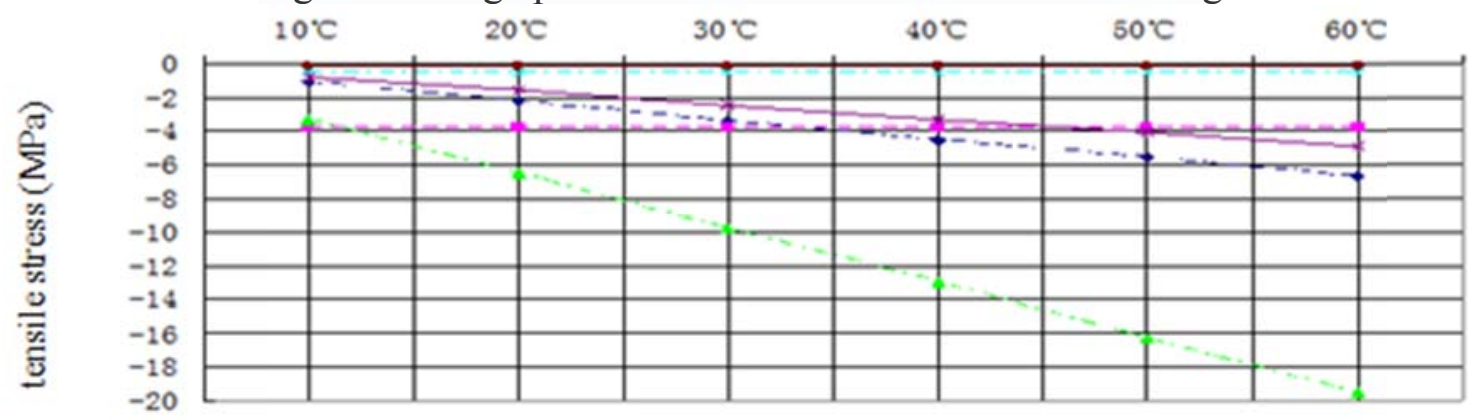

Cross-sectional temperature tensile stress

Temperature tensile stress at the bottom of the side slope Side span cross-sectional temperature tensile stress
Cross-sectional weight tensile stress

The gravity tensile stress of the bottom section of the side slope Side cross-sectional weight tensile stress

Fig 3 the bridge Tensile stress when the overall warming

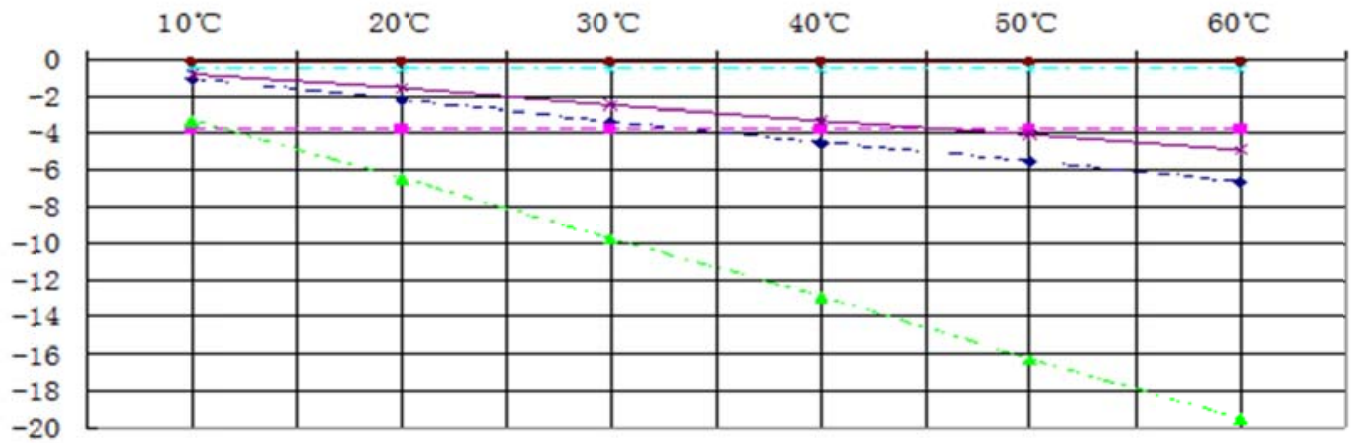

Cross-sectional temperature pressure stress

Cross-sectional weight pressure stress

Temperature pressure stress at the bottom of the side slope The gravity pressure stress of the bottom section of the side slope Side span cross-sectional temperature pressure stress

Side cross-sectional weight pressure stress

Fig 4 the bridge pressure stress when the overall cooling 


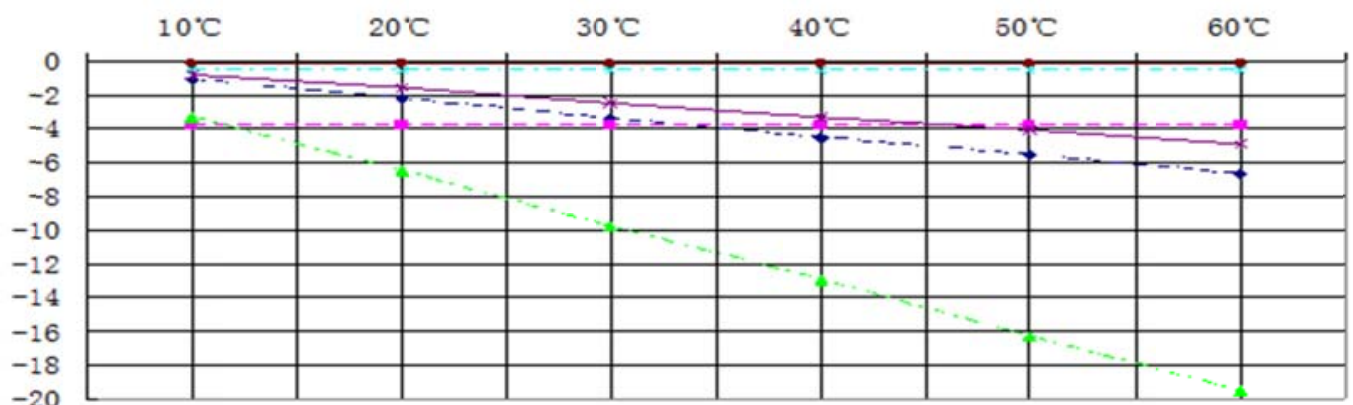

Cross-sectional temperature tensile stress

Temperature tensile stress at the bottom of the side slope Side span cross-sectional temperature tensile stress

Cross-sectional weight tensile stress

The gravity tensile stress of the bottom section of the side slope Side cross-sectional weight tensile stress

Fig 5 the bridge Tensile stress when the overall cooling,

By the analysis of the above four can be the diagonal rod, in the middle span and side span in the thermal stress and gravity stress:

(1) on the base of the cross section of the side slope, when the structural temperature rises or falls in the whole, the compressive stress increases remarkably, and the tensile stress increases remarkably. Far more than the weight of the side slope of the stress.

(2) in the cross section, when the temperature of the structure is increased, the compressive stress increases significantly, and the tensile stress increases significantly. When the temperature increases, the compressive stress will be greater than 33, and the tensile stress of the dead will always be greater than the tensile stress caused by the whole heating. When the whole cooling temperature is lower than 25 , the temperature stress will be greater than that of the gravity pull, while the temperature of the temperature is always less than that of the dead.

(3) on the cross section of the cross section, when the structural temperature rises, the temperature stress increases obviously and is always greater than the edge stress. The temperature tensile stress increases obviously at the temperature of 15 . When the temperature drops, the temperature stress increases obviously and is always greater than the edge stress, the temperature stress increases obviously and is always greater than the edge.

\section{Summary}

Through the analysis of engineering examples of this type of the bridge span beam, side span girder and the diagonal rod, respectively under uniform temperature field were stress analysis. The non abutment slant legged rigid frame bridge girder and the diagonal rod model in two kinds of temperature stress has considerable influence.

\section{Acknowledgements}

This work was financially supported by the National Science Foundation of China (51068012) a nd the Yunnan Province Communications DepartmentScience and Technology Program (2013(c)07 ) .

\section{References}

[1] Jin Wencheng,Gao Rong xiong Analysis of characteristics of non abutment slant legged rigid frame bridge[J] .road. 2000 ( 7):50-52

[2] Wang lin. Analysis of temperature and creep effect of large span prestressed concrete bridge [D]. Zhejiang University. 2005. 